\title{
LOS PERIODISTAS RECIÉN TITULADOS Y EL MERCADO LABORAL
}

\section{Introducción}

Una de las quejas más frecuentes en las salas de redacción de los medios de comunicación es sobre la deficiente calidad de las actuales cohortes de periodistas que llegan a hacer sus prácticas o a trabajar como colaboradores. Las críticas apuntan a que las nuevas generaciones carecen de las destrezas necesarias para un desempeño competitivo y a problemas que persisten desde la enseñanza media y que las universidades no superaron. Reproches parecidos existen en otras fuentes de empleo de periodistas.

La colisión entre las expectativas y la realidad surge también entre los periodistas jóvenes. Atraídos por la publicidad de las escuelas de periodismo, suelen tener altas expectativas de esta profesión. El choque con la realidad puede ser devastador cuando los jóvenes titulados constatan la realidad de jornadas laborales prolongadas, con frecuencia en malas condiciones, y la dificultad para conseguir trabajo. Entre los periodistas late cierto desencanto, no siempre manifiesto, por el efecto de la expansión del sistema universitario en la carrera, desencadenado por la reforma del sistema de educación superior en los años ochenta.

Fue esta realidad la que alentó la presente investigación sobre los recién titulados de periodismo y el mercado laboral. Esta carrera, que hasta 1973 era impartida por cuatro universidades, es hoy una de las más extendidas dentro de la educación superior. En 2005 la ofrecieron 36 universidades en 68 sedes, se matricularon en primer año 1.626 alumnos y se titularon 853. De profesión para elites, se masificó por el gran aumento de escuelas y programas universitarios que la imparten. En una década (1986-2005) las escuelas de periodismo han titulado 
7.709 alumnos, un volumen muy superior al total de los que trabajan en prensa en el país, número estimado en 1.713 , en 150 medios de comunicación, entre 2000 y 2001 (Gronemeyer, 2002).

Diversas evidencias indican que hay saturación en el mercado de los periodistas. La carrera, que en 1999 era la cuarta favorita de los postulantes a universidades, en 2005 no está entre las diez primeras. Los puntajes de acceso a periodismo bajaron 85 puntos en el último lustro y el número de titulados cayó 16,3\% en el trienio 2003-2005, desde 2002. El Colegio de Periodistas critica que las remuneraciones son bajas y el desempleo alto. En enero de 2007, su presidente, Luis Conejeros, sostuvo que "con 11 mil periodistas ya titulados y más de 8 mil estudiantes en 52 escuelas, los jóvenes deben ponderar muy bien la oferta educacional y la posibilidad real de lograr una inserción en el mundo laboral". El dirigente pidió a las universidades y al Ministerio de Educación transparentar información "imprescindible para que los jóvenes y sus familias puedan tomar decisiones adecuadas, en caso que quieran matricularse en carreras de Periodismo" (Colegio de Periodistas, 2007).

La información disponible sobre su mercado de trabajo es escasa, a pesar del papel esencial de estos profesionales en la sociedad. Además, se requiere mayor reflexión sobre los factores globales que inciden en este mercado y su evolución probable, para un mejor diseño curricular de la carrera. Hay cierto consenso de que, en un contexto de alto crecimiento e inserción internacional de la economía, los medios de comunicación social han experimentado fuertes mutaciones en las últimas dos décadas, debido a factores propios y exógenos de la industria. Entre los primeros resalta la tendencia a la concentración de los medios de comunicación social, debido al mayor volumen de inversión que requiere la competitividad y al déficit de políticas públicas que cautelen el pluralismo; entre los segundos, el avance tecnológico en las comunicaciones, que ha desplazado las audiencias, con el resultado de un aumento de la influencia de la televisión en detrimento de la prensa escrita y la radio, así como el surgimiento de medios digitales en internet y empresas de comunicaciones. La adaptación de los 
periodistas, la industria y la academia a estas nuevas condiciones ha sido compleja. Diversos estudios señalan debilidades en el desempeño de estos profesionales, entre ellas falta de autonomía e independencia (Gronemeyer, 2002), y cuestionan el carácter oligopólico del sistema de medios en el país, que afecta al pluralismo y la libertad de expresión (Corrales y Sandoval, 2005), lo que redunda, finalmente, en la calidad de la información que produce la prensa (Vera, 2001).

La investigación realizada para el Consejo Superior de Educación $(\mathrm{CSE})^{1}$ reunió la información de campo disponible sobre este mercado y acopió nuevos datos sobre la oferta y demanda de periodistas. A partir de una encuesta a profesionales del medio recién titulados y entrevistas cualitativas a ejecutivos de empresas e instituciones que los contratan, analizó los resultados y formuló algunas recomendaciones orientadas a mejorar la calidad de la enseñanza de esta carrera.

Este artículo, que resume algunos hallazgos de la citada investigación, se estructuró en seis secciones, además de esta introducción. La segunda sección se centra en la importancia de la información para los actores del sistema de educación superior. La metodología usada se describe en la tercera sección. El siguiente apartado presenta algunos indicadores del mercado laboral de los periodistas. En la quinta sección se examina el impacto ambivalente de las nuevas tecnologías en la empleabilidad de éstos. La sexta sección presenta las conclusiones del artículo y la séptima una reflexión sobre cómo mejorar la empleabilidad de estos profesionales.

\section{Marco de referencia}

La investigación busca mejorar la información sobre los titulados en periodismo. Entre las principales debilidades del sistema de educación superior está la falta de relación entre la oferta de carreras de pregrado de las universidades y la posibilidad real de encontrar empleo en el futuro

1 Délano, Manuel; Niklander, Karin y Susucasa, Paula (2007). La enseñanza del periodismo y el mercado laboral, investigación para el Consejo Superior de Educación (CSE). Santiago de Chile, mimeo. 
(Sepúlveda, 2005). Las distorsiones por la falta de correspondencia entre la oferta y la demanda de profesionales en pregrado, cuya corrección es imperiosa, implican fuertes costos humanos y sociales y constituyen un desafío pendiente para las políticas públicas.

Como señala un reciente estudio, "desde el punto del ajuste de la oferta y demanda, la falta de información en educación superior constituye un claro tipo de disfuncionalidad de sistema" (Zapata, 2006). Incluso, desde la perspectiva meramente económica, la toma de decisiones sin información dificulta el equilibrio en un mercado competitivo, como el de la educación superior, entre una masa de estudiantes que se orienta por el prestigio de las instituciones y las universidades, las cuales no siempre son transparentes.

Para algunos (Sepúlveda, 2005), su corrección implica más regulación del Estado, lo que puede significar, por ejemplo, fortalecer la acreditación e intervención directa para que haya una oferta de carreras coherente con las necesidades del país. Para otros (Brunner, 2006), existe un "abanico diversificado de dispositivos regulatorios (...) que dan lugar a un campo de disputas por la definición y el control de éstos". Entre estos dispositivos, Brunner menciona las encuestas estudiantiles de satisfacción, para conocer las percepciones y opiniones de los consumidores del sistema, y los informes sobre graduados, en los que sugiere avanzar más allá de los datos que recopila el observatorio "Futuro Laboral", e informar sobre los ingresos de los graduados de diferentes universidades identificadas, lo que transparenta resultados tal como los evalúa el mercado.

El papel de la información es relevante para la accountability del sistema universitario. La exploración que se presenta en estas páginas contribuirá a destacarla como uno de los soportes esenciales para una toma de decisiones adecuada (Dittborn y Aedo, 2005). El déficit de información o su mala calidad afecta a los estudiantes (insatisfacción y deserción), pero también a las universidades y al sistema en su conjunto, lo que, además, repercute en las empresas que contratan a estos profesionales. 
Mejorar la información sobre la oferta y demanda de periodistas favorecerá a todos los actores del sector. A los estudiantes, para una mejor toma de decisiones al momento de resolver a qué carrera postular, con el fin de que la decisión contemple tanto su vocación como la realidad del mercado laboral; a las universidades, que podrán adaptar sus mallas de formación a las características del mercado; y a las empresas e instituciones que contratan periodistas, para conocer la formación que se impartió a los titulados.

En materia de información en la educación superior, se requiere mejorar el acceso, pertinencia, descentralización y diversidad, validez y confiabilidad, comparabilidad, calidad, universalidad, coordinación y cooperación, como apunta Zapata (2006). La información es, por sí misma, un mecanismo de regulación que ayuda a los estudiantes a decidir dónde y qué carrera estudiar, a los empleadores a resolver mejor el perfil de egresados que necesitan, a las universidades a realizar una gestión más adecuada y a mejorar la calidad de sus servicios, a las políticas públicas a determinar mejor las carencias y demandas, y a los investigadores como insumo (Zapata, 2006).

\section{Metodología}

El estudio, efectuado en el segundo semestre de 2006 y comienzos de 2007, reunió y sistematizó la información disponible sobre la oferta de periodistas, sus perfiles y sesgos, y la demanda laboral en los campos en que, a grandes rasgos, las universidades los preparan para desempeñarse: periodismo noticioso, comunicaciones, producción y gestión de medios, y docencia e investigación. Por necesidades de síntesis, este último aspecto de la investigación no se presenta en este artículo.

Posteriormente, se realizaron dos estudios de campo en el marco de esta investigación. El primero fue una encuesta estructurada a una muestra aleatoria y representativa de 141 recién titulados de periodismo, en su mayor parte en 2005, de ambos sexos y de universidades del Consejo de Rectores y autónomas ubicadas en cuatro de las cinco regiones del país que reúnen a un mayor número de titulados de esta 
carrera (Región Metropolitana y II, V y VIII regiones). Contestaron 41 preguntas sobre su educación y la de sus padres, postulación a la universidad, formación universitaria recibida, práctica profesional, situación en el mercado laboral y vínculos trabajo-universidad.

El segundo fue cualitativo y se realizó entre octubre de 2006 y enero de 2007 mediante entrevistas semiestructuradas a 15 directores, editores y ejecutivos (en adelante, "ejecutivos") de medios de comunicación, empresas y reparticiones estatales que son importantes fuentes de empleo de periodistas, para analizar la demanda laboral de estos profesionales: cuatro diarios (tres nacionales y uno regional); dos radios de alcance nacional; dos canales de televisión; cuatro empresas de comunicaciones (dos en Santiago y dos en regiones), y tres representantes de áreas de comunicaciones de reparticiones de los poderes Ejecutivo, Legislativo y de un organismo autónomo del Estado. Previamente se entrevistó a representantes del Colegio de Periodistas y del observatorio "Futuro Laboral".

La representatividad de estos entrevistados es elevada. Los ejecutivos son de empresas e instituciones que contratan más de 530 periodistas y sobre 650 si se incluyen los colaboradores. Varios de estos medios y empresas son líderes de su sector y los entrevistados son profesionales de trayectoria.

\section{Algunos indicadores del mercado de periodistas}

El observatorio "Futuro Laboral" indica que el número total de periodistas es de 10.769 (www.futurolaboral.cl), cifra con la que coincide el presidente del Colegio de Periodistas, que estima en 11.000 estos profesionales (Colegio de Periodistas, 2007).

"Futuro Laboral" y el Colegio de Periodistas no tienen información sobre empleo y desempleo de estos profesionales, aunque esta última entidad estima que entre 70\% y $80 \%$ "está desempleado o cumpliendo funciones que no son propias de lo que estudió"2.

2 Entrevista con los autores en junio de 2006. 
Según datos del Servicio de Impuestos Internos (SII), en 2004 y 2005 presentaron su declaración anual de renta 3.467 periodistas cada año. Aunque esto no refleja empleo, indica que casi un tercio de los periodistas declara impuestos como tal.

Suponiendo que el número anual de periodistas titulados siguiera constante en 850 (fueron 853 en 2005, según el CSE) y considerando que existe un universo de 10.769 , si este sector tuviera una tasa de retiro por pensiones de vejez e invalidez similar a la de los trabajadores afiliados a las AFP en 2005 (0,42\% de 7.350 .591 personas) ${ }^{3}$ se puede concluir que el número de periodistas se duplicará el 2019 (tabla 1). Según este ejercicio, el número de periodistas aumenta inicialmente $7,8 \%$ y termina con un incremento anual de $3,2 \%$, porque los titulados anuales son constantes mientras crece el total de titulados. En 2005 se retiraron 45 periodistas para pensionarse por vejez e invalidez, y 110 lo harán en 2025.

Tabla 1. Proyección de número total de periodistas por año, 2005-2025

\begin{tabular}{c|c|c|c}
\hline Años & $\begin{array}{c}\mathrm{N}^{0} \text { total de } \\
\text { periodistas }\end{array}$ & $\begin{array}{c}\mathrm{N}^{0} \text { de periodistas } \\
\text { titulados por año }\end{array}$ & $\begin{array}{c}\mathrm{N}^{o} \text { de periodistas } \\
\text { pensionados por año }\end{array}$ \\
\hline 2005 & 10.769 & 850 & 45 \\
\hline 2006 & 11.574 & 850 & 49 \\
\hline 2007 & 12.375 & 850 & 52 \\
\hline 2008 & 13.173 & 850 & 55 \\
\hline 2009 & 13.968 & 850 & 59 \\
\hline 2010 & 14.759 & 850 & 62 \\
\hline 2011 & 15.547 & 850 & 65 \\
\hline 2012 & 16.332 & 850 & 69 \\
\hline 2013 & 17.113 & 850 & 72 \\
\hline 2014 & 17.891 & 850 & 78 \\
\hline 2015 & 18.666 & 850 & 82 \\
\hline 2016 & 19.438 & 850 & 85 \\
\hline 2018 & 20.206 & 850 & 98 \\
\hline 2019 & 20.971 & 850 & 94 \\
\hline 2020 & 21.733 & 850 & 98 \\
\hline 2021 & 22.492 & 850 & 101 \\
\hline 2022 & 23.248 & 850 & \\
\hline
\end{tabular}

3 La proyección no considera la variable de tasa de mortalidad. 


\begin{tabular}{l|l|l|l}
\hline 2023 & 24.749 & 850 & 104 \\
\hline 2024 & 25.495 & 850 & 107 \\
\hline 2025 & 26.238 & 850 & 110 \\
\hline
\end{tabular}

Fuente: Elaboración de los autores, basada en datos del Consejo Superior de Educación y Superintendencia de AFP.

\section{Impacto de las nuevas tecnologías en el empleo de periodistas}

La empleabilidad de los periodistas preocupa también más allá de las fronteras del país. La revolución de las tecnologías de la información a un ritmo vertiginoso en los últimos decenios se ha expresado en tendencias opuestas en el empleo de estos profesionales. Por una parte, el proceso global de fusiones, absorciones y concentración internacional de los medios de comunicación social tiende a reducir el número de empleos y su calidad. Por otra, se abre para ellos el nuevo territorio ocupacional de la industria multimedia, en la cual se multiplica la difusión del conocimiento bajo diversas formas y hay una creciente convergencia de sectores antes separados, como los de informática, telecomunicaciones, editoriales y espectáculos con los medios de comunicación (OIT, 2000).

Entre los periodistas, el fenómeno suele ser observado más como amenaza que como oportunidad, porque perciben que los empleadores les aumentan el trabajo con las nuevas tecnologías, mientras estos últimos argumentan que las nuevas destrezas los hacen más empleables.

Para los periodistas independientes, el desarrollo tecnológico se ha traducido, en general, en ventajas que les permiten elevar su productividad, debido a la mayor rapidez y el menor costo de las comunicaciones, la facilidad y oportunidad de acceso a la información, y la simplificación de las correcciones y edición, que pueden compensar los costos del equipamiento. En cambio, para los periodistas de plantilla, la sobrecarga de información es una queja frecuente. El uso de internet y de bases de datos, y las comunicaciones a través del correo electrónico se han generalizado en las salas de redacción 
del mundo, generando tanto un flujo continuo de información a los medios -y por consiguiente un aumento de la carga de trabajo- como una disminución de los derechos de autor.

La OIT sostiene que "nadie está en condiciones de dar una respuesta definitiva" a si las tecnologías de la información crean o destruyen empleo en los medios de comunicación. La evidencia en países desarrollados muestra que en algunos campos ha aumentado el empleo (producción de contenidos), mientras que en otros ha disminuido (tipógrafos, maquetistas, técnicos de sonido, camarógrafos). Los nuevos puestos de trabajo creados por el uso innovador de la tecnología, sin embargo, "suelen caracterizarse por su inestabilidad" (OIT, 2000).

Proyecciones del Ministerio del Trabajo de Estados Unidos reflejan que el empleo de analistas en medios, periodistas y corresponsales crecerá menos que el promedio de otras ocupaciones hasta 2014. Influirán en ello la consolidación y convergencia de la industria de los medios y teledifusión, así como el desarrollo tecnológico, aunque igualmente la creación de nuevos medios requerirá un mayor número de estos profesionales (U.S. Department of Labor, 2007).

Aunque cabría esperar un continuo incremento del número de ocupaciones para periodistas -considerando que muchos aspectos esenciales de su trabajo no pueden ser automatizados y que las nuevas tecnologías permiten el surgimiento de empresas en el ciberespacio, creando más empleos-, diversas evidencias muestran que los diarios y revistas han ido perdiendo público en la mayor parte de los países (Ramonet, 2005), y sobre estos profesionales pende el temor a la sustitución de los medios tradicionales por los electrónicos.

En América Latina, investigaciones de los sindicatos afiliados a la Federación Nacional de Periodistas del Brasil (FENAJ) señalan que ha disminuido el número de profesionales en las redacciones, mientras que se ha elevado el volumen y ritmo de trabajo. Un asesor técnico del Sindicato de Periodistas de Sao Paulo afirma que los profesionales recién egresados de las universidades superan "en mucho" el número de puestos de trabajo creados anualmente en los 
medios de comunicación, lo que unido a quienes pierden su empleo en las redacciones, implica mayor desempleo. Esto ha facilitado a las empresas la exigencia de cumplir horas extraordinarias que después no se remuneran (De Almeida, 1998). El aumento del empleo ocurre en empresas pequeñas y medianas, que tienen poco personal, y en el trabajo independiente. Las grandes empresas tienden a reducir costos y subcontratar labores que antes eran parte de su actividad básica.

En suma, el impacto de las nuevas tecnologías en el empleo de los periodistas, que en Chile también concita atención, tiene matices. Constituye todavía un proceso en desarrollo cuyos resultados serán más evidentes con la maduración de las tendencias aquí señaladas.

\section{Conclusiones}

La comparación de los resultados obtenidos con los dos instrumentos de campo permitió obtener una visión panorámica de este mercado laboral para los periodistas recién titulados. De toda la información obtenida, se presenta a continuación una síntesis de algunos de los principales resultados.

\section{a. Mercado laboral}

- Alta oferta y baja demanda de periodistas recién titulados Las escuelas de periodismo entregaron al mercado cerca de 900 periodistas titulados anuales entre 2000 y 2005, nueve veces más que la demanda anual, que el presidente del Colegio de la Orden (2007) sitúa entre 80 y 100 profesionales. Si bien los titulados podrían disminuir en el futuro porque bajó el ingreso (de 2.247 en 2003 a 1.649 en 2005), existe un claro desequilibrio entre oferta y demanda de éstos.

La opinión de la mayoría de los 15 ejecutivos entrevistados coincide, y algunos incluso dicen que hay "saturación" en el mercado.

4 Todas las citas de ejecutivos incluidas en esta sección fueron tomadas de la transcripción de las entrevistas que se les realizaron en la investigación para el CSE. Véase Délano, Niklander y Susacasa (2007). 
Uno de ellos sostiene que un estudiante de periodismo tiene hoy mucho menos asegurado un trabajo que en el pasado. Otro critica la incapacidad del Estado para regular la oferta de periodistas, lo que generará "cesantes ilustrados (...) un joven que entra a estudiar periodismo pensará que el Estado un día lo va a salvar y lamentablemente no lo salvará". Un tercer ejecutivo advierte que el mercado chileno es muy pequeño, con una industria de medios muy consolidada, y considera muy probable que en el futuro no haya mucho más trabajo del actual. Otro afirma que existe un "divorcio entre las necesidades reales del mercado y la cantidad de ofertas de escuelas", en el que las universidades consideran la demanda de quienes quieren estudiar esta carrera, pero no "la demanda real de periodistas".

De acuerdo con los ejecutivos, la rotación de periodistas es "bajamedia", lo que implica un mercado con escasa movilidad, en especial en los diarios, televisión y el Estado, siendo más heterogénea la situación en las empresas de comunicaciones y "media" en las radios.

\section{- Alto desempleo de periodistas recién titulados}

La alta oferta y baja demanda se han traducido, primero, en elevado desempleo de los recién titulados. En el estudio se midió el desempleo como periodista, es decir el no ejercicio de la profesión por el encuestado. Si bien este criterio es elástico, ya que alguien podría preferir voluntariamente no ejercer la profesión y no porque el mercado se lo impone, el volumen de desempleo encontrado debilita esa eventual objeción.

En el ámbito nacional, el 32,9\% de los periodistas recién titulados no tiene empleo en su profesión (gráfico 1), más en regiones (36,2\%) que en Santiago (31,2\%). Este nivel de desempleo casi quintuplica la tasa de desocupación nacional que el Instituto Nacional de Estadísticas (INE) calculó para el trimestre enero-marzo de 2007. Para encontrar magnitudes sobre $30 \%$ en el país es preciso retroceder hasta la crisis de comienzos de los años ochenta. El resultado es consistente con la estimación de los ejecutivos entrevistados: 14 de 15 estimaron que el desempleo de los periodistas es superior al promedio nacional y cinco sobre $50 \%$. 
Gráfico 1. Periodistas recién titulados de Santiago y de las regiones II, V y VIII: ¿tiene trabajo como periodista?

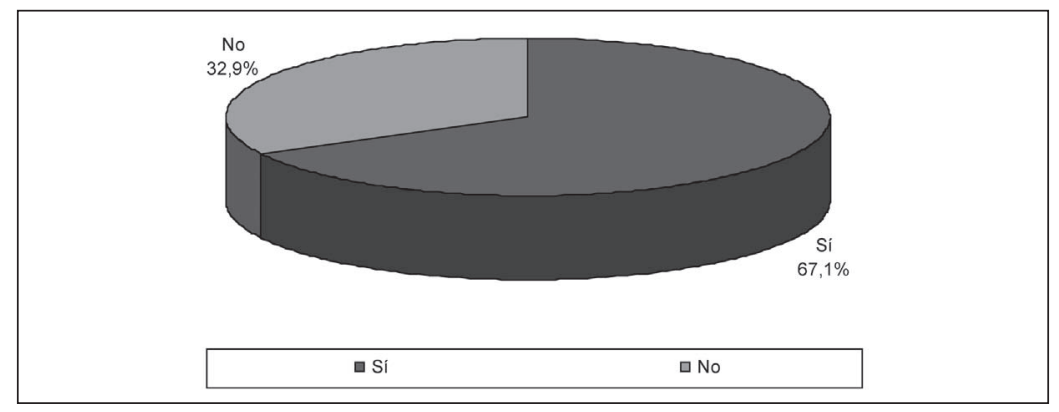

Si bien parte del desempleo puede explicarse porque los recién titulados todavía no se insertan en el mercado, 40\% tiene más de seis meses buscando trabajo como periodista y $15 \%$ sobre un año $(26,3 \%$ en regiones). El 31,7\% no ha buscado empleo como periodista, lo que podría interpretarse como fuerza de trabajo desalentada.

De este resultado se desprende que uno de cada cinco periodistas recién titulados $(20,1 \%)$ no tiene ningún tipo de trabajo. De los que están desempleados como periodistas, el 44,4\% tiene otro tipo de trabajos, que en su mayoría - no todos- pueden considerarse subempleo por calificación. Al pedírsele a los encuestados especificarlo, se obtuvieron estas respuestas, entre otras: productor manager de vestuario infantil, secretaria, asesor de seguros, expositor en charlas informativas sobre Transantiago*, vendedor de tienda comercial, docente universitario, promotor, asistente de marketing, de talleres de capacitación, guía turístico, administrador de restaurante, de departamento de cobranzas, de hostería. Esto puede ser indicativo de la dificultad para conseguir trabajo como periodista, pero también de que es posible obtener mejores remuneraciones o condiciones en otras tareas.

El ejercicio de empleos no periodísticos de baja calificación por parte de recién titulados podría significar una inversión personal, familiar y social perdida en formación profesional. La carrera dura

* Sistema de transporte implementado este año por el Estado chileno. 
cinco años en la mayoría de las universidades y el precio de la matrícula oscila entre $\$ 90.000$ y más de $\$ 200.000$, mientras que el costo del arancel anual varía entre un mínimo de $\$ 1,75$ millones y un máximo de \$3,25.

Un ejecutivo sostiene que las universidades entregan "periodistas a la sociedad que no tienen dónde ejercer y de repente terminan haciendo cualquier cosa menos periodismo". Otro plantea que, probablemente, el desempleo de los periodistas es más alto que el promedio nacional "porque no tienen herramientas para desarrollarse en otras áreas". Si por ejemplo él fuera un agente de banco, "¿por qué voy a contratar a un periodista? (...) El campo es muy estrecho”.

\section{- Trabajo precario}

Entre los periodistas recién titulados existe subempleo. Casi uno de cada diez $(9,5 \%)$ trabaja uno o dos días a la semana como periodista y el 23,3\% no cumple una semana laboral completa (gráfico 2). Esto es coherente con que el 40,4\% de ellos tiene trabajos secundarios (pitutos) como periodista (43,9\% en Santiago y 33,3\% en regiones). Esta información es consistente con la visión de los ejecutivos entrevistados y del Colegio de Periodistas.

Gráfico 2. Periodistas recién titulados de Santiago y de las regiones II, V y VIII: horas de trabajo a la semana como periodista

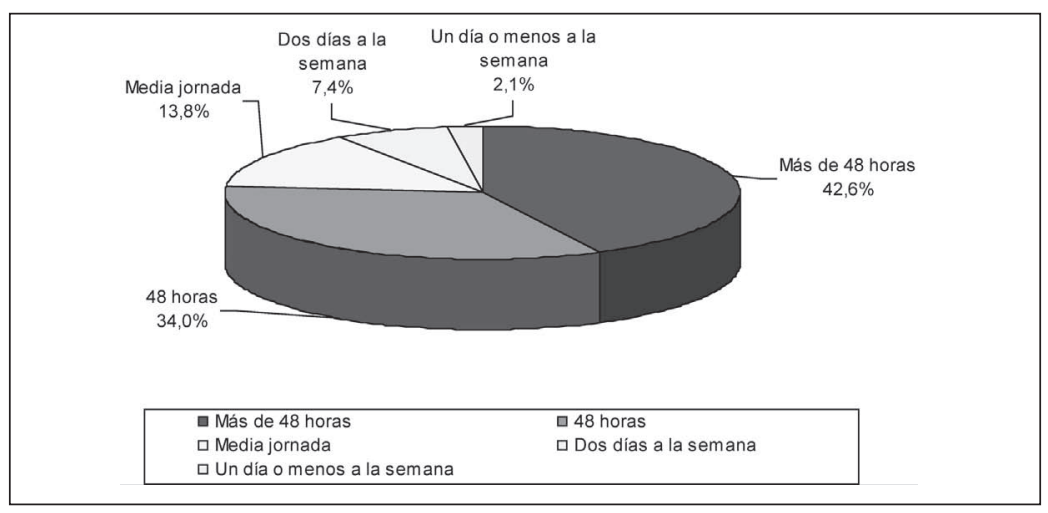


También se observan jornadas laborales muy prolongadas: $42,6 \%$ de los periodistas recién titulados señala trabajar sobre 48 horas a la semana (48,4\% en regiones). Aunque esta situación es generalizada en la sociedad chilena, no implica necesariamente lograr mayor productividad, según estudios de organismos internacionales.

De los recién titulados con empleo en su profesión, el 37,7\% no tiene ningún tipo de contrato laboral. Un tercio (32,7\%) tiene contrato indefinido, el $6,1 \%$ a plazo fijo y un $23,5 \%$ a honorarios.

De los periodistas recién titulados con empleo en su profesión, el empleador sólo paga previsión, salud y colación al 13,6\%. Aunque el $42,6 \%$ tiene jornadas muy extensas, sólo a 4\% le pagan más por las horas extraordinarias. El beneficio más extendido es el aguinaldo de Navidad o Fiestas Patrias (20,6\%) (gráfico 3). Los ejecutivos entrevistados señalaron que en sus empresas e instituciones se paga previsión, salud y otros beneficios a los periodistas contratados.

Gráfico 3. Santiago y regiones II, V y VIII: beneficios que el empleador paga al periodista recién titulado \%

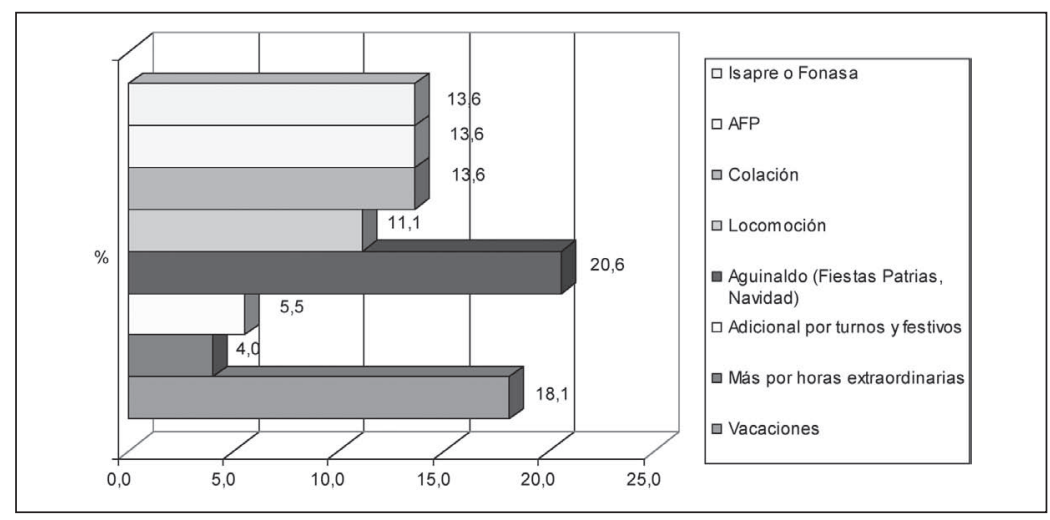

- Remuneraciones

El 64\% de los periodistas recién titulados tiene en su empleo principal remuneraciones brutas inferiores a $\$ 400.000$. En Santiago el 56,1\% está similar situación, pero en regiones el 80,6\%, lo que implica una importante brecha. El 9,3\% señala ganar menos de $\$ 100.000$ brutos 
y un $24,8 \%$ bajo $\$ 200.000$; en contraste, el $5,2 \%$ tiene ingresos sobre $\$ 700.000$ brutos y el $15,6 \%$ sobre $\$ 500.000$ (gráfico 4).

Gráfico 4. Periodistas recién titulados de Santiago y de las regiones II, V y VIII: remuneración bruta promedio mensual, por tramos, del trabajo principal (\%)

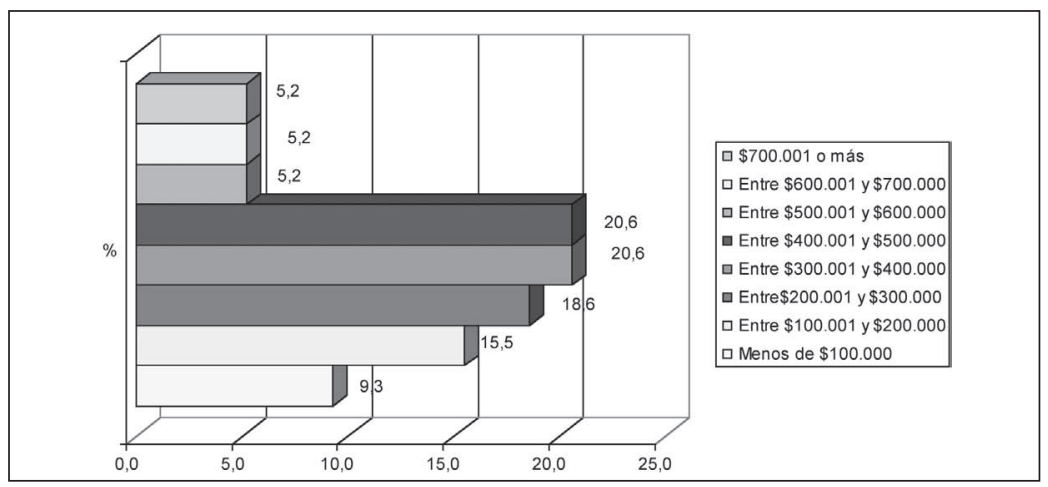

Al considerar la remuneración bruta mensual total de los periodistas recién titulados se reduce la concentración en los tramos bajos de ingresos. El $56,7 \%$ tiene ingresos totales bajo $\$ 400.000$ brutos (gráfico 5). En Santiago se concentran en estos tramos el $48,3 \%$ y en regiones el $76 \%$. La remuneración bruta de los que tienen contrato indefinido tiene un descuento de salud y previsión (20\%), y la de aquellos con honorarios el pago de impuestos (10\%).

Gráfico 5. Periodistas recién titulados de Santiago y de las regiones II, V y VIII: remuneración bruta promedio mensual total, por tramos

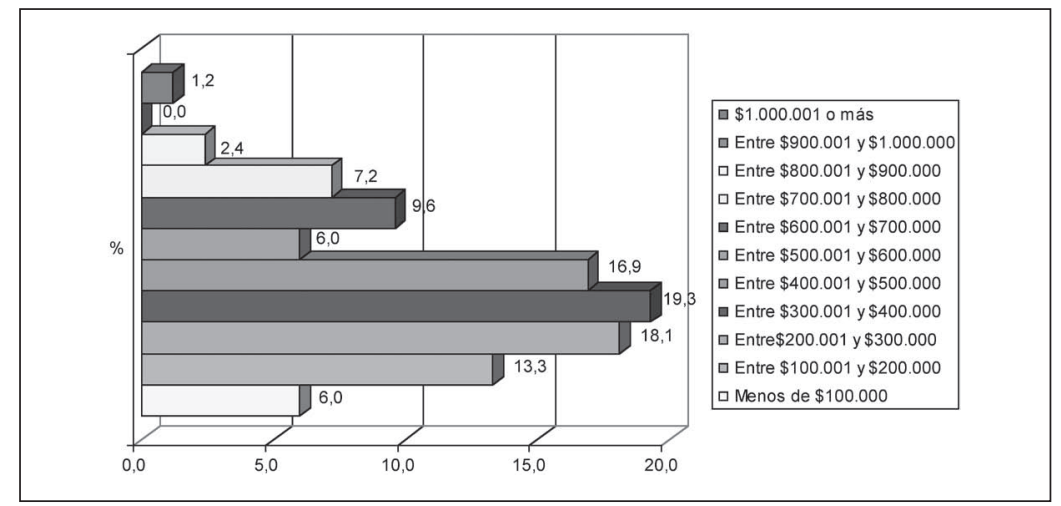


Los ejecutivos entrevistados indican que los periodistas recién titulados tienen ingresos líquidos de $\$ 418.000$ en promedio. Esto es equivalente a remuneraciones brutas de $\$ 522.500$ y superiores a las que señalan los encuestados. Esto no es extraño pues debe considerarse que ellos encabezan empresas o instituciones líderes en sus respectivos ámbitos.

El observatorio "Futuro Laboral" informa de remuneraciones brutas promedio para los periodistas, dos años después de titulados, de $\$ 494.541$, y de $\$ 585.805$ con cuatro años de trabajo. Al descontar el 20\% de seguridad social, la información es consistente con lo que señalan los encuestados, en promedio. Sin embargo, debe recordarse que los promedios pueden ocultar situaciones extremas: por ejemplo, uno de cada cuatro $(24,8 \%)$ recién titulados tiene remuneraciones brutas mensuales bajo $\$ 200.000$.

- Diferencias entre Santiago y las regiones II, V y VIII

La encuesta permitió corroborar la impresión de los ejecutivos entrevistados respecto de que las condiciones de trabajo de los periodistas recién titulados son más precarias en las regiones II, V y VIII que en Santiago. Así lo señalaron los encuestados en la gran mayoría de los indicadores (empleo, condiciones de trabajo, remuneraciones). Esto puede atribuirse a que el mercado laboral es más pequeño en esas regiones y a la consiguiente menor disponibilidad de fuentes de trabajo. Varios ejecutivos resaltaron la mayor precariedad del trabajo en regiones, que hace a los periodistas "más vulnerables a las fuentes".

- Campos de ejercicio profesional

Tanto la encuesta como la investigación previa ratificaron los campos de ejercicio profesional más conocidos (medios, Estado y comunicaciones), pero en las entrevistas varios ejecutivos indicaron nuevas áreas de empleo posibles para la profesión. Uno de ellos señaló la importancia de que los periodistas sepan desarrollar emprendimientos personales como, por ejemplo, "un taller de periodismo en un colegio". Otro apuntó a las agencias de publicidad 
como un nuevo espacio de empleo, algo que las universidades no han advertido. También sostuvo que los periodistas podrían desempeñarse en recursos humanos, por la centralidad que tienen las comunicaciones en la administración o en la generación de políticas públicas.

\section{b. Formación universitaria}

- Diferencias entre las visiones de ejecutivos entrevistados y periodistas recién titulados

En los resultados del trabajo de campo resaltaron las diferencias entre la visión crítica de los ejecutivos entrevistados sobre los recién titulados y la buena valoración de estos últimos acerca de las escuelas de periodismo. Salvo el reconocimiento generalizado de los ejecutivos sobre la buena formación de los recién titulados en materia de nuevas tecnologías, hubo pocas valoraciones positivas. Según señalaron los ejecutivos, éstos presentan, en general, problemas de redacción, ortografía y reporteo, baja lectura, falta de nivel cultural, de conocimientos de actualidad, de capacidad reflexiva y de especialización, así como poco manejo del idioma inglés (tabla 2). 


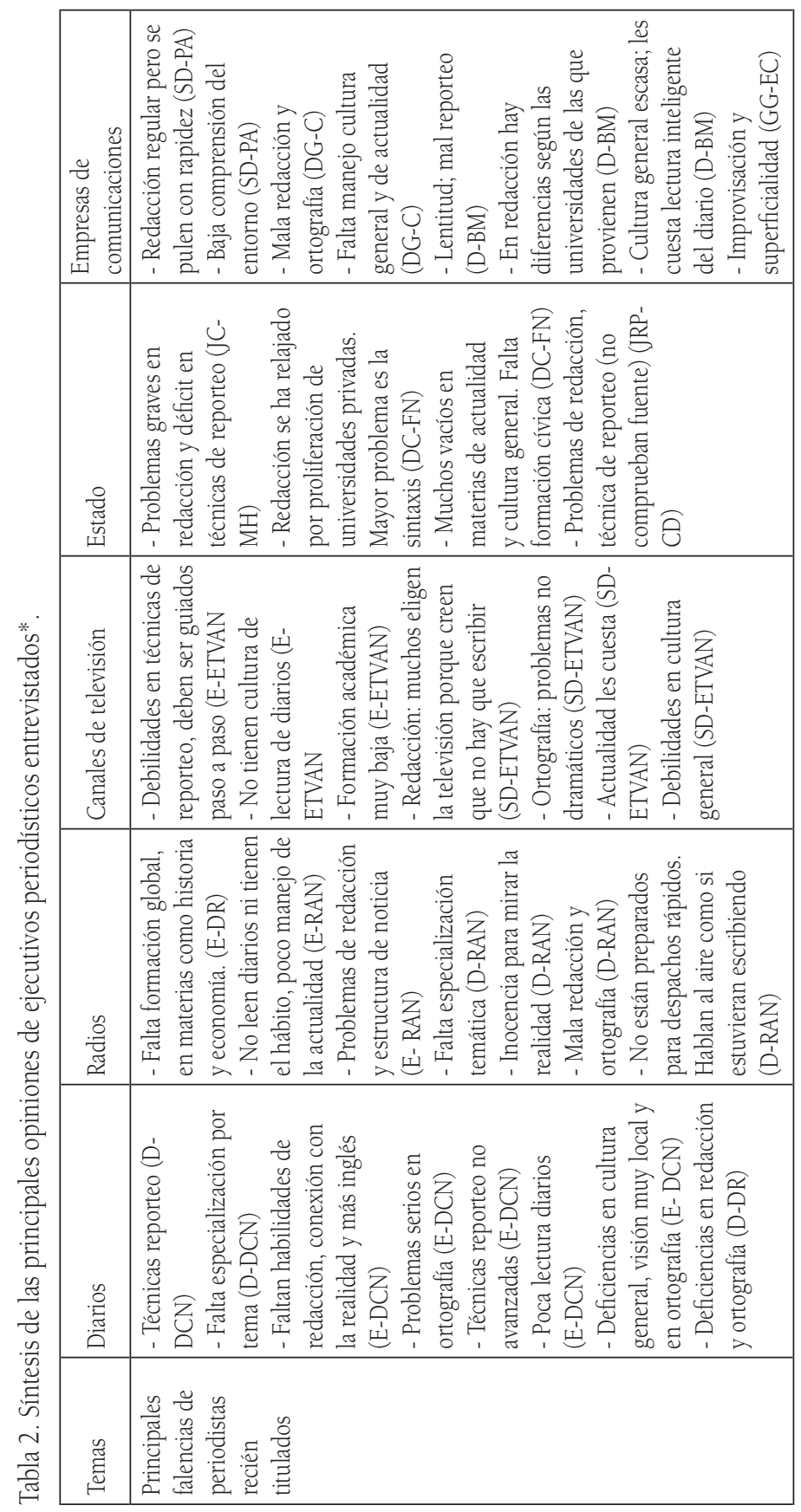


224 PERIODISTAS RECIÉN TITULADOS Y MERCADO LABORAL - Manuel Délano, Karin Niklander, Paula Susacasa

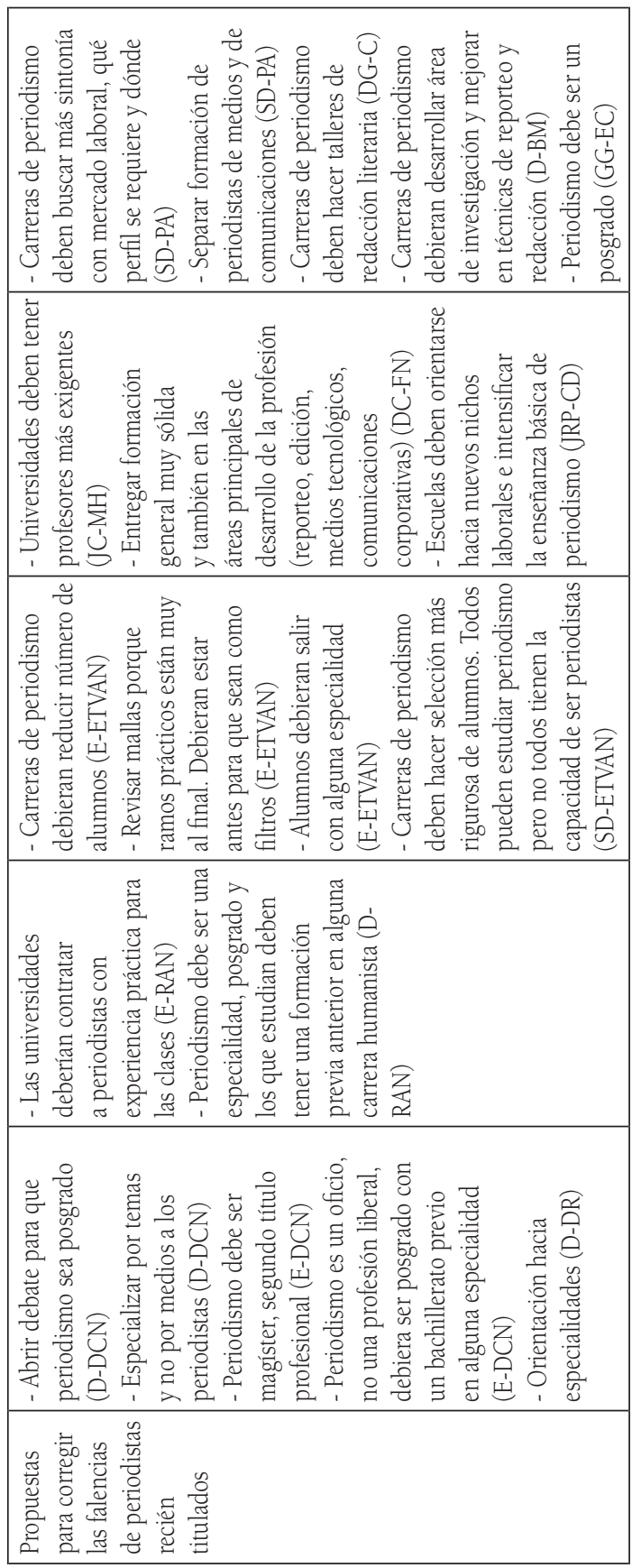




\begin{tabular}{|c|}
\hline 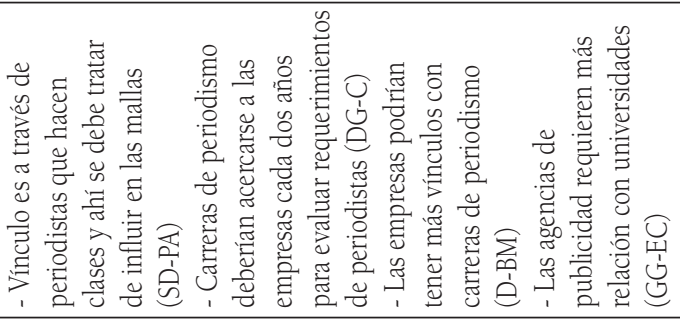 \\
\hline 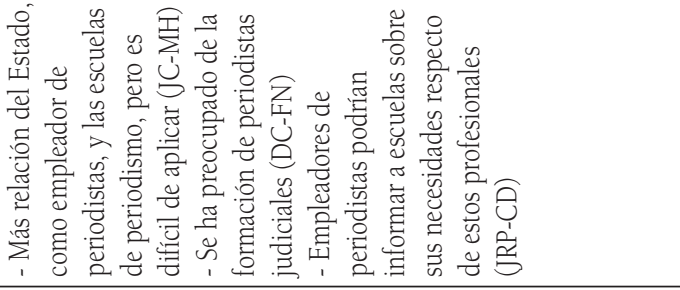 \\
\hline 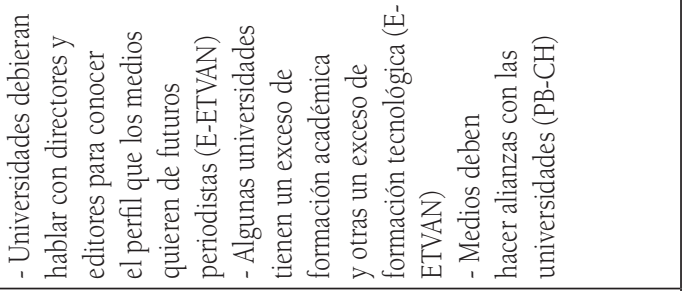 \\
\hline 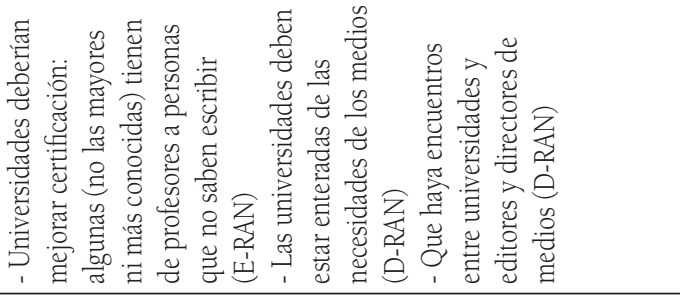 \\
\hline 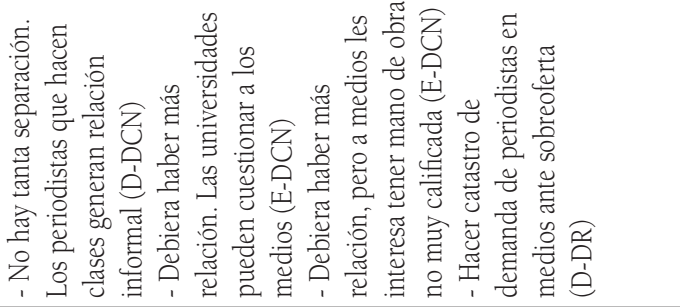 \\
\hline 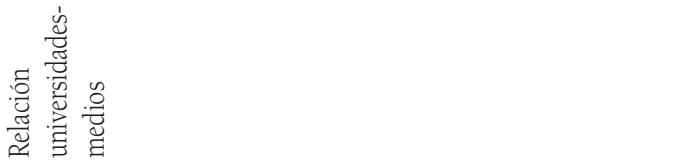 \\
\hline
\end{tabular}


226 PERIODISTAS RECIÉN TITULADOS Y MERCADO LABORAL - Manuel Délano, Karin Niklander, Paula Susacasa

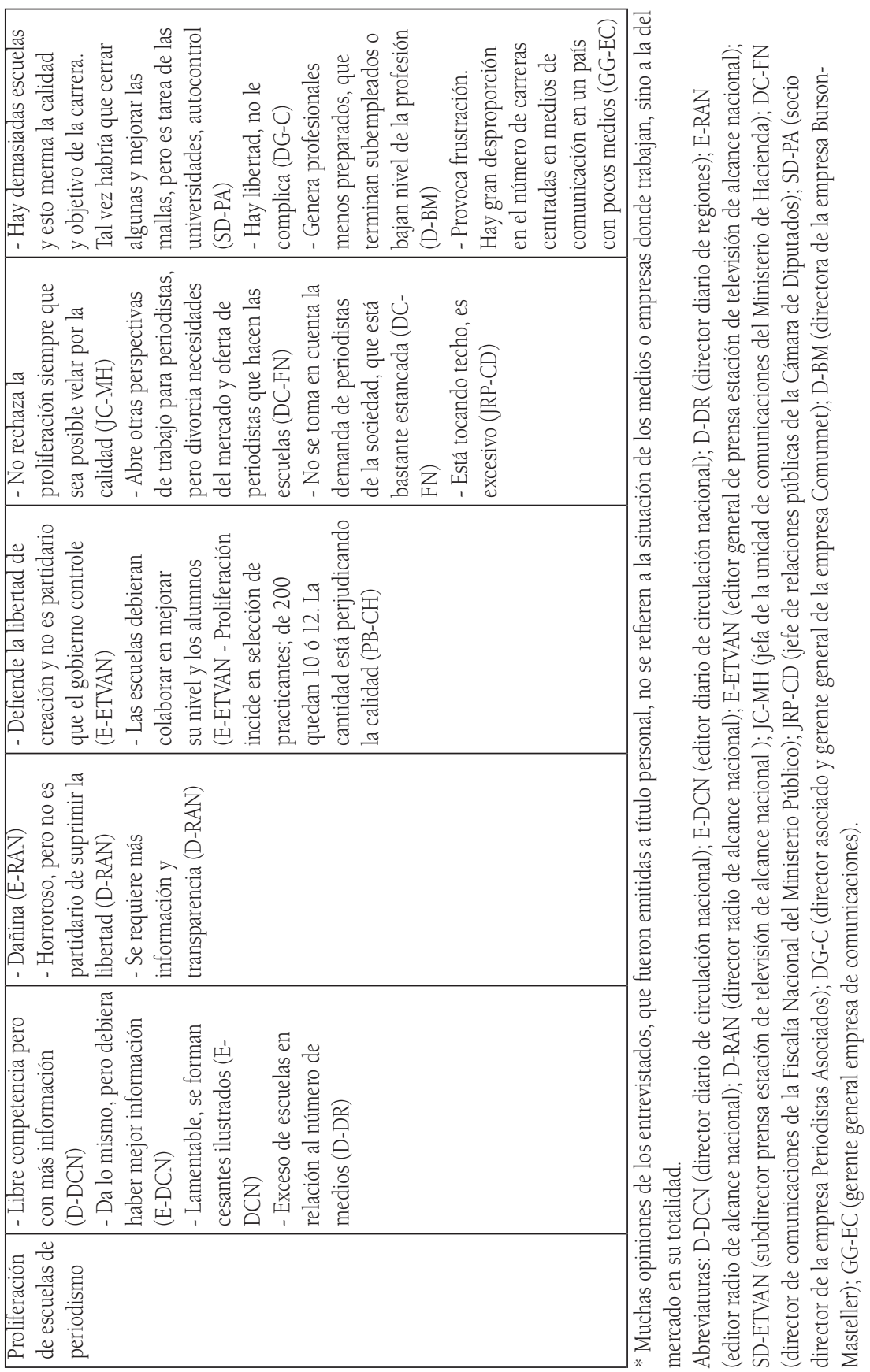


Los periodistas recién titulados, en cambio, sostienen casi unánimemente (99,2\%) que su práctica fue evaluada como "excelente" o "buena". Sin embargo, sólo el 44,0\% permaneció trabajando o colaborando en el lugar donde hizo la práctica, porcentaje que informa mejor de la real evaluación, dado que constituye un criterio importante para contratar.

Los juicios de los ejecutivos entrevistados sobre las falencias de los periodistas recién titulados fueron, en general, lapidarios. Uno de ellos criticó su falta de habilidades de redacción, porque afecta la productividad al distraer a los editores de su trabajo, "que es pensar". Otro afirmó que les cuesta acercarse a un hecho noticioso y traducirlo en un texto. Un tercero sostuvo que llegan con ímpetu de grandes escritores, pero se pierden al construir una frase. Un cuarto entrevistado dijo que no es habitual ver "a un muchacho joven leyendo el diario de verdad, cuando más los titulares y las portadas de los diarios electrónicos y nunca viendo el detalle", lo que incide después en la pobreza de contenidos de sus notas. Otro reprochó que los reporteros nuevos "son un poquito las personas preparadas para ir a la conferencia de prensa y sacar la cuña". Un sexto apuntó que su principal falencia es la "improvisión o falta de reflexión" al escribir.

- Relación entre la universidad y las empresas que emplean periodistas

Para los periodistas recién titulados, la primera relación entre la universidad y el mercado laboral se produce cuando acuden a las empresas o instituciones a realizar su práctica profesional. El 33,3\% sostuvo que consiguió su práctica profesional con ayuda de la universidad y el resto con sus redes personales. El origen de la ayuda fue heterogéneo: $40 \%$ el diario mural, 25\% al postular a la práctica, 20\% los profesores, $10 \%$ el sistema de orientación y $5 \%$ los contactos.

Al preguntarles a los recién titulados con trabajo cómo lo consiguieron, $43 \%$ sostiene que fue por buen desempeño en la práctica. El 60,8\% considera que la universidad no incidió favorablemente al conseguir empleo; esto sugiere que, aparte de los 
profesores que trabajan en medios, empresas e instituciones, existe poca relación entre la academia y el mercado, algo que corroboran los ejecutivos entrevistados.

Al momento de contratar periodistas, la gran mayoría de los ejecutivos dijo no tener preferencias por algún tipo de universidades. En ocho de las empresas o instituciones es requisito poseer título de periodista para ser contratado y en seis no lo es; de estas últimas, en tres los ejecutivos prefieren igualmente un titulado.

Algunos ejecutivos entrevistados admitieron sesgos. Uno planteó que, en general, vienen más capacitados los periodistas de universidades a las cuales es más difícil ingresar. Otro reconoció un sesgo hacia los que provienen de las universidades de Chile (UCH) y Pontificia Católica de Chile (PUC); un tercero lo amplió a las que, a su juicio, tienen más formación básica-UCH, PUC, de Santiago de Chile (USACH) y de Concepción-. Otro entrevistado resaltó la formación académica en televisión de los recién titulados de la PUC, seguidos por los de la Universidad Diego Portales. Pero otros ejecutivos discreparon: uno dijo que de todas las universidades salen periodistas buenos y malos. Otro, que hay diferencias pero no según los propietarios de cada universidad. Un tercero afirmó que se advierten diferencias por experiencia o cursos de postítulo.

- Proliferación de escuelas de periodismo

Aunque todos los ejecutivos entrevistados admitieron una proliferación, discreparon sobre sus efectos y la forma de enfrentarla (tabla 2). Para algunos no constituye un problema, siempre que los postulantes reciban más y mejor información sobre el mercado laboral y sea posible velar por la calidad de las escuelas de periodismo. La mayoría es proclive a que haya libertad y rechaza una intervención directa del Estado en el tema.

Para otros, la proliferación genera profesionales menos preparados que terminan desempleados o bajando el nivel de la profesión, provocando frustración y bajas remuneraciones. Uno 
expresó que "la cantidad está perjudicando la calidad" y otro que tal vez debieran cerrarse algunas escuelas y mejorar las mallas, demandando, a su vez, "autocontrol" a las universidades.

Hubo mayor consenso en que debe haber mayor información y transparencia y en que las escuelas deben mejorar su calidad.

- Reconocimiento del periodismo

A los ejecutivos se les preguntó si es efectivo que hay un menor reconocimiento de la carrera de periodismo - dada la baja en el puntaje de ingreso a la carrera y el número de postulantes- y cuáles son las eventuales causas de esta merma. La mayoría admitió la validez de esta aseveración, pero tres de ellos la negaron o relativizaron.

Varios apuntaron como causas las bajas remuneraciones y el exceso de periodistas. Uno de ellos culpó a la confusión entre las industrias del entretenimiento y de las noticias, lo que se traduce en la intromisión de la farándula y la decadencia programática de la televisión y algunos medios escritos. Otro lo atribuyó a que es fácil titularse de periodista. Un tercero indicó que esta profesión nunca ha tenido reconocimiento, porque la ejercen personas que no son periodistas.

- Malla de las carreras de periodismo

El 70,3\% de los periodistas recién titulados afirmó que conocía la malla de la carrera antes de ingresar (57,8\% en regiones). En general, los encuestados valoran positivamente las asignaturas que tuvieron. El 75,4\% afirmó que tuvo un porcentaje de ramos útiles superior al 38,1\% del total y sólo el 24,5\% sostuvo que menos de la mitad le fue útil. La preparación de sus profesores fue considerada "buena" o "excelente" por el 66,5\% de los recién titulados, aunque con una brecha de 31,9 puntos porcentuales entre Santiago $(77,4 \%)$ y regiones $(45,5 \%)$.

En cambio, siete de los 15 ejecutivos entrevistados afirmaron no conocer las mallas de las carreras en las que estudiaron los periodistas recién contratados y varios de quienes las conocían señalaron que éstas no inciden en la definición de contratos. Los que conocen o han 
revisado las mallas fueron críticos de éstas. Uno de ellos las calificó de "superficiales", porque los estudiantes no reciben suficiente formación; otro criticó la debilidad en los ramos de formación general. Un tercero afirmó que tienen poca innovación y son muy tradicionales, mientras un cuarto las calificó de "desactualizadas".

\section{Reflexión final}

Los resultados de ambos estudios de campo reflejan, en general, un fuerte desajuste entre la oferta y demanda de periodistas recién titulados, cuyos principales problemas son el desempleo, subempleo y precariedad de condiciones, en mayor medida en Santiago que en regiones, lo que además refleja una brecha según ubicación geográfica. La información resultó ser consistente, en gran medida, con las evidencias parciales de otras fuentes, como el Colegio de Periodistas, estudios académicos y el observatorio "Futuro Laboral", aunque esta investigación se refiere sólo a la generación que está incorporándose recién al mercado de trabajo y no a la totalidad de estos profesionales.

Desajustes de este nivel (uno de cada cinco recién titulados está desempleado y uno de cada tres no ejerce su profesión) en otros sectores han ameritado la aplicación de programas de empleo por parte del Estado. Sin embargo, la situación de estos periodistas recién titulados no lo requiere, porque el 65,5\% vive con sus padres y sólo uno de cada cinco $(21,1 \%$ ) vive solo o con su pareja, mayoritariamente con apoyo familiar. Por otro lado, tampoco su situación es asimilable a la del desempleo juvenil, dado que uno de cada tres encuestados $(72,3 \%)$ tiene entre 25 y 29 años de edad.

En términos más generales, considerando que el número de periodistas se duplicaría hacia el 2019, versus un mercado que no prevé para este sector una expansión de puestos de trabajo y en el que constituye todavía una incógnita el impacto de las nuevas tecnologías en el empleo, el desajuste entre oferta y demanda compete centralmente a las escuelas de periodismo. Evidencias preliminares recogidas con posterioridad a esta investigación indican que en algunas 
de estas escuelas se están elevando las exigencias académicas y existe preocupación por la amplitud del campo profesional. En cualquier caso, la mejoría de la empleabilidad de los periodistas debiera constituir un indicador fundamental de la calidad de la enseñanza entregada por la universidad. Una primera tarea prioritaria para las escuelas que aún no lo hacen es recoger y sistematizar información comparable sobre los profesionales que están titulando, lo que debiera ser estimulado por el CSE y también por el Colegio de Periodistas.

La proliferación de escuelas de periodismo requiere de la regulación y el contrapeso que significaría contar con información veraz, oportuna y adecuada del mercado, para que los postulantes conozcan la realidad laboral que encontrarán al egresar y no se enteren sólo al terminar sus estudios. Al postular a periodismo, un alumno de cuarto medio y su familia debieran estar en condiciones de evaluar el monto de inversión en los cinco años de duración promedio y el eventual retorno que tendrá el egresado. Sin embargo, como el acceso a la información es asimétrico (es más accesible para los grupos de mayores ingresos que para los más vulnerables), como garante del bien común en el sistema de educación superior, el CSE debiera también brindar información y velar por su disponibilidad para todos. Si el mercado no es capaz de lograr esta autorregulación, sería necesario dotar al Estado de instrumentos que ordenen la oferta de profesionales y no esperar que haya una crisis para intervenir, porque para muchas familias la inversión en la educación de sus hijos es la mayor esperanza de progreso intergeneracional.

Junto con demandar información fidedigna bajo criterios uniformes a las carreras (dadas las inconsistencias encontradas en esta investigación entre la del CSE y la que entregan las universidades), se podría avanzar hacia el diseño de un ranking por carreras, partiendo por periodismo, con criterios que permitan medir calidad de la enseñanza, tal como existe en forma incipiente en el sector privado. Al CSE le cabe un papel más proactivo en este campo que en la actualidad.

El mejoramiento de la calidad de la enseñanza del periodismo es una segunda iniciativa que elevaría la empleabilidad de sus titulados. 
Un primer paso en esa dirección sería que sólo las universidades acreditadas por el CSE puedan entregar títulos profesionales en el caso de esta carrera. Otro, que depende de las universidades, es que esta carrera deje de ser un pregrado y se transforme en un magíster -como proponen varios de los ejecutivos entrevistados-, considerando la demanda del mercado por mayores grados de especialización para la comprensión de fenómenos cada vez más interrelacionados. El conocimiento y manejo instrumental que requiere la interpretación de las transformaciones sociales, económicas, políticas y en otras disciplinas -tarea central de los periodistas- requiere de más tiempo que el necesario para la enseñanza de las técnicas periodísticas. Este modelo permitiría mejorar y diversificar la oferta de los profesionales que titulan las escuelas de periodismo, quienes accederían al mercado con una licenciatura y una maestría. La mejor calificación de los periodistas debería incidir en elevar sus remuneraciones.

Estrechamente relacionado con lo anterior, las escuelas de periodismo deberían aumentar sus esfuerzos de actualización y difusión de sus mallas, dado el desconocimiento y las críticas que sufren en el mercado laboral. La especialización en áreas del conocimiento donde hay necesidades informativas - que se anticipan crecientes en el futuro- contribuiría a mejorar la empleabilidad de los recién titulados. Una alternativa que implicaría igualmente un avance sería la especialización en ciertas áreas de la profesión.

Las universidades podrían subir el puntaje mínimo de ingreso a periodismo y a otras carreras donde se advierta saturación del mercado. Esto permitiría mejorar la calidad de los profesionales y reducir la matrícula de ingreso. Igualmente, agregar una prueba especial de acceso facilitaría la selección de los postulantes, tal como ya hacen algunas carreras.

Un tercer ámbito en el que se pueden emprender esfuerzos para mejorar la empleabilidad de los periodistas es el estrechamiento de relaciones entre la academia y el mercado. Tanto las escuelas como las empresas que contratan estos profesionales obtendrían ventajas 
mutuas de los vasos comunicantes. A las primeras, les facilitaría el conocimiento de las tendencias futuras del mercado y la adaptación de las mallas y asignaturas, lo que favorecería la autorregulación de la oferta de recién titulados; a las segundas, les permitiría informar a las escuelas del perfil de profesionales que necesitan. Adicionalmente, las carreras requieren mantener registros y seguimiento de la trayectoria de sus egresados y titulados.

A los periodistas les compete un papel protagónico en este proceso. Tendrían que ser los principales interesados en mejorar la empleabilidad de su profesión. En este terreno, el Colegio de Periodistas puede también desempeñar un papel importante.

Por último, la metodología de este estudio, que combina un instrumento cuantitativo y otro cualitativo para contrastar la opinión de recién titulados y de quienes los contratan, podría ser adaptada para aplicarse en otras profesiones donde también existen síntomas de saturación del mercado laboral. El acopio de esta información facilita la toma de decisiones para enfrentar problemas de mercado en áreas específicas.

\section{Referencias bibliográficas}

Brunner, José Joaquín. (2006) Mercados universitarios: ideas, instrumentaciones $y$ seis tesis en conclusión. (Sitio en Internet) Disponible en http:// mt.educarchile.cl/mt/jjbrunner/archives/2006/05/post_9.html

Colegio de Periodistas de Chile. (2007) Piden mayor transparencia en mercado de escuelas de periodismo. (Sitio en Internet) Disponible en http://www.colegiodeperiodistas.cl/index.php?action=actualidad_ nacional\&noticia_id=159

Corrales, Osvaldo y Sandoval, Juan. (2005) Concentración del mercado de los medios, pluralismo y libertad de expresión. Documentos de Investigación, Instituto de la Comunicación e Imagen, Centro de Estudios de la Comunicación, Universidad de Chile. (Sitio en Internet) Disponible en: http://www.comunicacion.uchile.cl/corrales2005.html

De Almeida, Carlos Alberto. (1998) La convergencia tecnológica y algunos de sus impactos sobre los trabajadores en el Brasil. En La convergencia 
multimedia: romper el silencio, Educación Obrera 1998/1-2, Núm. 110/111, OIT. (Sitio en Internet) Disponible en http://www.ilo.org/ public/english/dialogue/actrav/publ/110/led110.htm

Délano, Manuel; Niklander, Karin; Susucasa, Paula. (2007) La enseñanza del periodismo y el mercado laboral. Investigación para el Consejo Superior de Educación (CSE), Santiago de Chile: mimeo.

Dittborn, Paulina y Aedo, Cristián. (2005) Oferta, demanda y políticas públicas en educación superior. Calidad en la Educación, 22, pp. 55-68.

Futuro Laboral. Chile. Carreras y mundo del trabajo. (Sitio en Internet) Disponible en http://www.futurolaboral.cl/FuturoLaboral/DetalleCarreras

Gronemeyer, María Elena. (2002) Periodistas chilenos: El reto de formar profesionales autónomos e independientes. Cuadernos de Información, (15), pp. 53-70.

OIT (2000) Las tecnologías de la información en las industrias de los medios de comunicación y del espectáculo: Sus repercusiones en el empleo, las condiciones de trabajo y las relaciones laborales. Documento base para el Coloquio sobre las Tecnologías de la Información en las Industrias de los Medios de Comunicación y el Espectáculo, 28 febrero al 3 de marzo 2000, Ginebra, OIT.

Ramonet, Ignacio. (2005) Los medios de comunicación en crisis. Le Monde Diplomatique en Español, 111, enero, pp. 1 y 26-27.

Sepúlveda, Cecilia. (2005) Cambios en la demanda por programas profesionales de pregrado y el desafío que ello impone a las políticas públicas y la universidad. Calidad en la Educación, 22, pp. 71-78.

U.S. Department of Labor, Bureau of Labor Statistics. (2007) Occupational Outlook Handbook (OOH), 2006-07 Edition. (Sitio en Internet) Disponible en http://www.bls.gov/oco/home.htm

Vera, Héctor. (2001) Relación entre la calidad de la información en Chile y la formación en las escuelas de periodismo. Ponencia en el Encuentro Nacional Enseñanza del Periodismo en Chile y Campo Laboral, ASEPECS, septiembre 2001, Santiago de Chile. (Sitio en Internet) Disponible en http://www.periodismo.uchile.cl/asepecs/

Zapata, Gonzalo. (2006) Información en Educación Superior. Calidad en la Educación, 24, pp. 197-213.

Recibido: 2 de agosto de 2007

Aceptado: 29 agosto de 2007 